\title{
Low temperature magnetic properties and stress effects in glassy Fe-B alloys: the eutectic region
}

\author{
A. B. Beznosov, E. L. Fertman, V. V. Eremenko, V. A. Desnenko, and \\ V. Z. Bengus
}

B. Verkin Institute for Low Temperature Physics and Engineering, National Academy of Sciences of Ukraine, 47, Lenin Ave., 310164, Kharkov, Ukraine

E-mail: beznosov@ilt.kharkov.ua

Received May 26, 1999

\begin{abstract}
The magnetic properties of glassy $\mathrm{Fe}_{100-x} \mathrm{~B}_{x}(x=14,16,17,20)$ alloys under a low mechanical stress (up to $68 \mathrm{MPa}$ ) at temperatures $10-300 \mathrm{~K}$, in magnetic fields up to 40 Oe, have been studied. Low temperature anomalies of magnetic susceptibility, as well as substantial differences in the effects of stresses on the low field magnetic characteristics of alloys with various boron contents, have been revealed. The eutectic alloy $\mathrm{Fe}_{83} \mathrm{~B}_{17}$ was found to be the least sensitive to applied stresses, magnetic fields, and temperature changes: it possesses the lowest magnetic induction in the reversibility fields region and the highest local anisotropy fluctuations. The hypoeutectic $\mathrm{Fe}_{84} \mathrm{~B}_{16}$ alloy is the most sensitive to applied stresses at room temperature, but its sensitivity substantially reduces with increasing load, as well as with decreasing temperature. The results obtained reflect the special character of the alloys electronic structure at the eutectic point.
\end{abstract}

PACS: $75.30 . \mathrm{Cr}, 75.30 . \mathrm{Gw}, 71.23 . \mathrm{Cq}$

The amorphous alloys $\mathrm{Fe}_{100-x} \mathrm{~B}_{x}$ are soft magnetic materials with a wide field of applications [13]. In particular, much attention has focused on the alloys in the region of $x=17$ (the eutectic composition), due to the possibility of special interatomic interactions in these alloys [4,5]. We have used the effect of stress on the magnetic properties to study the nature of differences of the alloys properties.

\section{Experimental procedure}

The studied samples $\mathrm{Fe}_{100-x} \mathrm{~B}_{x}(x=14,16,17$, 20) were prepared from $30 \mu \mathrm{m}$ thick and $6 \mathrm{~mm}$ wide amorphous ribbons, obtained by the planar flow casting technique.

The effect of tension as well as pressure on the magnetic induction $B$, was measured. The pulse-induction technique was used, with longitudinal magnetic fields $0 \leq H \leq 40$ Oe, temperatures 300 and $77 \mathrm{~K}$, and tension stresses $\sigma$ near 22 and $63 \mathrm{MPa}$ in the 1st kind experiments, as well as the SQUID magnetometer, normal to the ribbons surface external magnetic field 20 Oe (the effective field in the samples was substantially reduced by the demagnetization factor $\quad N \sim 10$ ), temperatures
$10 \mathrm{~K} \leq T \leq 230 \mathrm{~K}$, and the normal to the ribbons surface uniaxial pressure of about $20 \mathrm{MPa}$ in the 2nd kind experiments.

\section{Results and discussion}

\section{Magnetic characteristics}

Longitudinal field. It was revealed that the eutectic alloy $\mathrm{Fe}_{83} \mathrm{~B}_{17}$ possesses the lowest value of $B$ in fields above 5 Oe at all studied temperatures and stresses. This is in agreement with the opinion, that fluctuations of the local anisotropy field in this alloy are the highest in the considered system [6]. The $B(H)$ curves (Fig. 1) suggest that the $\mathrm{Fe}_{83} \mathrm{~B}_{17}$ alloy has the smallest saturation induction. This result corresponds to the saturation magnetization measurements of Bengus et al. [4].

At $300 \mathrm{~K}$ the hypoeutectic alloy $\mathrm{Fe}_{84} \mathrm{~B}_{16}$ possesses the highest magnetic permeability $\mu$, and the lowest coercivity $H_{c}$ and magnetic remanence $B_{r}$ (Table). At $77 \mathrm{~K}$ the difference between the $\mu$ of $\mathrm{Fe}_{84} \mathrm{~B}_{16}$ and other alloys decreases. 


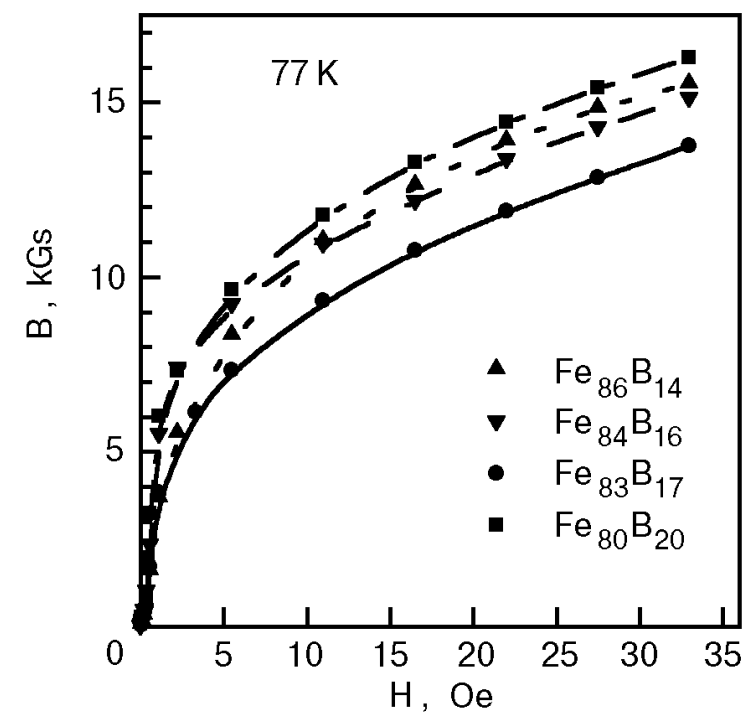

Fig. 1. The magnetic induction field dependences of $\mathrm{Fe}_{100-x} \mathrm{~B}_{x}$ $(x=14,16,17,20)$ amorphous alloys under zero load at $77 \mathrm{~K}$.

Table

The maximum magnetic permeability $\mu_{\max }$, coercivity $H_{c}$, and magnetic remanence $B_{r}$ of glassy Fe-B alloys at nitrogen $(77 \mathrm{~K}$ ) and room $(300 \mathrm{~K})$ temperatures.

\begin{tabular}{l|c|r|r|r|r|r}
\hline \hline \multirow{2}{*}{ Alloy } & \multicolumn{2}{|c|}{$\mu_{\max }$} & \multicolumn{2}{c|}{$B_{r}, \mathrm{Gs}$} & \multicolumn{2}{c}{$H_{c}, \mathrm{Oe}$} \\
\cline { 2 - 7 } & $77 \mathrm{~K}$ & $300 \mathrm{~K}$ & $77 \mathrm{~K}$ & $300 \mathrm{~K}$ & $77 \mathrm{~K}$ & $300 \mathrm{~K}$ \\
\hline \hline $\mathrm{Fe}_{86} \mathrm{~B}_{14}$ & 2957 & 7228 & 980 & 1358 & 0.495 & 0.231 \\
$\mathrm{Fe}_{84} \mathrm{~B}_{16}$ & 4600 & 12651 & 303 & 756 & 0.165 & 0.132 \\
$\mathrm{Fe}_{83} \mathrm{~B}_{17}$ & 3235 & 7478 & 419 & 1496 & 0.341 & 0.264 \\
$\mathrm{Fe}_{80} \mathrm{~B}_{20}$ & 3802 & 8357 & 1566 & 1804 & 0.418 & 0.308 \\
\hline \hline
\end{tabular}

Transversal field. The effective magnetic susceptibility of the samples $\chi_{\mathrm{eff}}$ measured in the 2nd experiment slowly decreased with decreasing temperature almost in the whole temperature range. There was a sharp slope at about $20 \mathrm{~K}$, which was evidently connected with a magnetic phase transition to the disordered asperomagnetic phase [7]. The $\chi_{\text {eff }}$ curve of the eutectic alloy $\mathrm{Fe}_{83} \mathrm{~B}_{17}$ was substantially lower than that of the other alloys. This may be considered as an evidence of the highest local magnetic anisotropy in the alloy.

Local anisotropy. The analysis of the field dependence of magnetization $\mathbf{M}=(\mathbf{B}-\mathbf{H}) / 4 \pi$ in the region approaching saturation is carried out in the framework of the local anisotropy model. The parameters $A_{i}$ of the equation

$$
M=\sum_{i=0}^{6} A_{i} H^{-0.5 i}
$$

in the region of longitudinal magnetic fields 16 Oe $\leq H \leq 40$ Oe were evaluated by the least

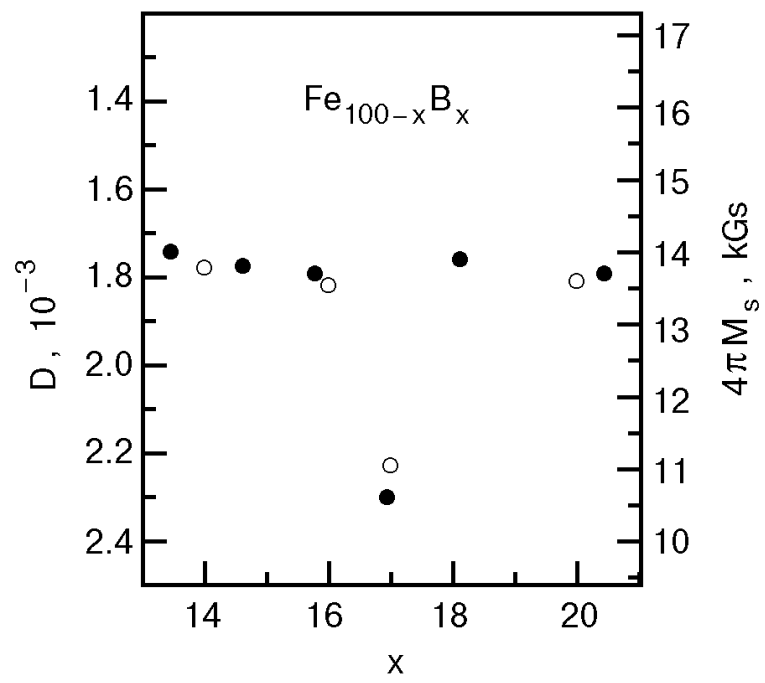

Fig. 2. The parameter $D(\bigcirc)$ from Eq. (2), reflecting the local magnetic anisotropy fluctuations in the amorphous alloys $\mathrm{Fe}_{100-x} \mathrm{~B}_{x}$ under zero load at $77 \mathrm{~K}$, and saturation magnetization (๑) [4] at $4.2 \mathrm{~K}$ versus $x$.

squares method. The validity was analyzed of the sum (1) as a whole, as well as of its separate components. It was found that the best approximation of experimental data corresponds to the equation

$$
M=M_{\infty}\left[1-\left(D M_{\infty} / H\right)^{0.5}\right],
$$

describing the zero-dimensional violations of the translation symmetry [8] of the system. The parameter $D$ in Eq. (2) characterizes the local magnetic anisotropy fluctuations value.

As one can see in Fig. 2, the concentration dependence of parameter $D$ shows a singularity at the eutectic point $x=17$, which correlates well with the analogous singularity of the low temperature high field magnetization $M_{s}$ from [4].

The results obtained agree with work by Iskhakov et al. [6], who reported that the eutectic alloy had the maximum value of mean square fluctuations of local magnetic anisotropy.

\section{Tension effects}

Applied tensile stresse leads to a growth in magnetic induction $B$, magnetic permeability $\mu$ and remanence $B_{r}$, and to a decrease in $H_{c}$ (but no $H_{c}$ decrease for $\mathrm{Fe}_{84} \mathrm{~B}_{16}$ ).

Permeability. The increase of magnetic induction is mainly seen in fields up to about 30 Oe. At both room and liquid nitrogen temperatures, the growth of the maximum value of magnetic permeability $\mu_{\max }$ of all the studied alloys under load is very sharp and range up to $1.5-3$ times at about 


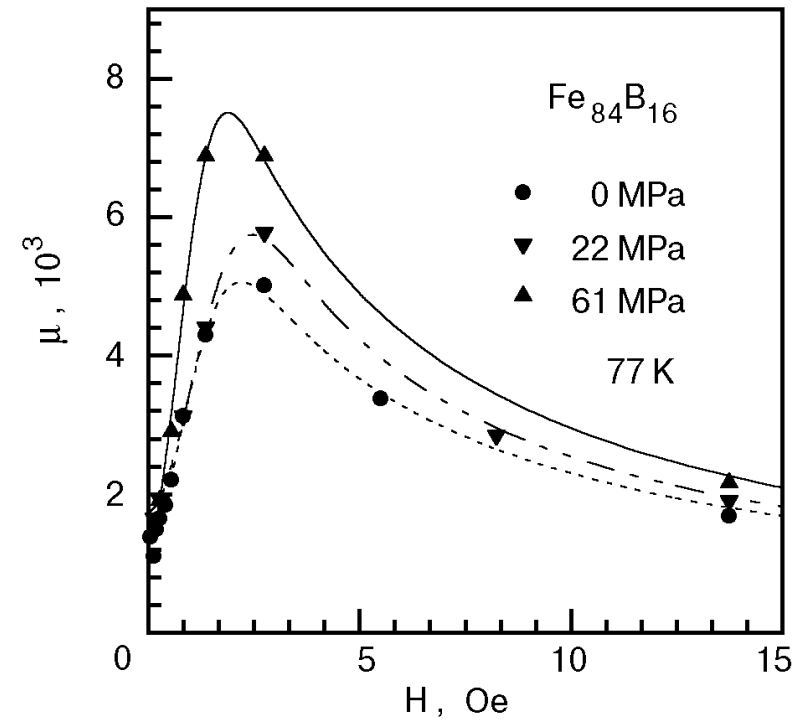

Fig. 3. Magnetic permeability $\mu$ of amorphous $\mathrm{Fe}_{84} \mathrm{~B}_{16}$ alloy at liquid nitrogen temperature under the various tensile loads (22 and $61 \mathrm{MPa}$ ) versus magnetic field $H$.

$63 \mathrm{MPa}$. This can be seen at Fig. 3, where the load effect is shown for the alloy $\mathrm{Fe}_{84} \mathrm{~B}_{16}$.

At $300 \mathrm{~K}$ the smallest value of $\Delta \mu_{\max } / \Delta \sigma$ is exhibited by the eutectic alloy $\mathrm{Fe}_{83} \mathrm{~B}_{17}$. When the temperature is lowered to $77 \mathrm{~K}, \mu_{\max }$ decreases about 2 times. The smallest value of $\Delta \mu_{\max } / \Delta \sigma$ at $77 \mathrm{~K}$ is for the $\mathrm{Fe}_{84} \mathrm{~B}_{16}$ alloy.

Magnetic remanence. The applied loads lead to 1.6-3.6 times growth in remanence; the change in remanence per unit stress $\Delta B_{r} / \Delta \sigma$, is nearly equal for all studied alloys and temperatures, except for the hypoeutectic alloy $\mathrm{Fe}_{84} \mathrm{~B}_{16}$. This alloy exhibits

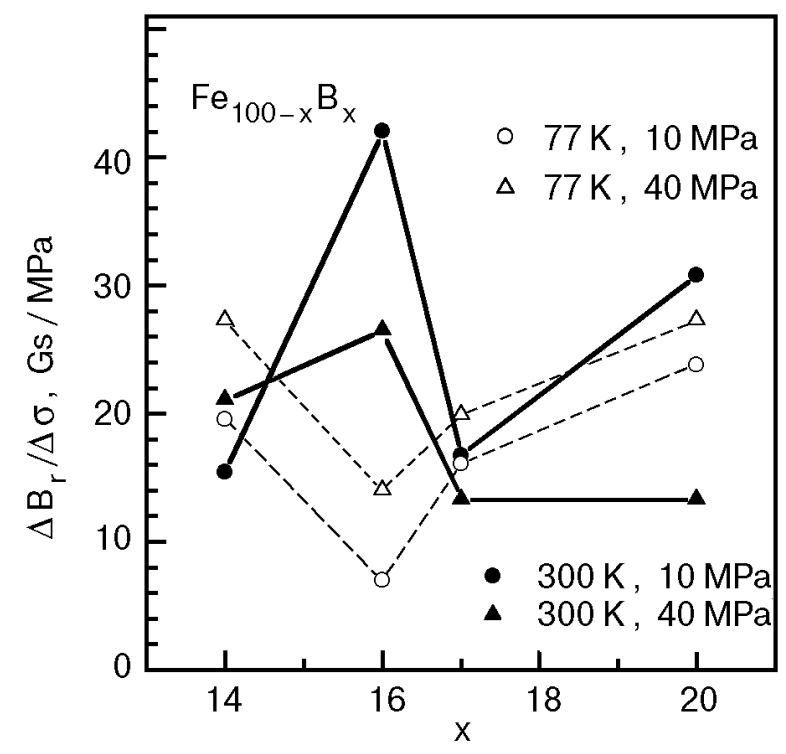

Fig. 4. The average derivative $\Delta B_{r} / \Delta \sigma$ of amorphous Fe-B alloys at temperatures 77 and $300 \mathrm{~K}$ under tension stresses of 10 and $40 \mathrm{MPa}$. the smallest remanence without load at both studied temperatures, the largest value of $\Delta B_{r} / \Delta \sigma$ at $300 \mathrm{~K}$ and the smallest at $77 \mathrm{~K}$ (Fig. 4).

Coercivity. The coercivity $H_{c}$ of all the alloys decreased with increasing applied load, except for $\mathrm{Fe}_{84} \mathrm{~B}_{16}$, which increased. But its coercivity remains the smallest at all the studied temperatures and loads. At the same time the coercivity of the eutectic alloy $\mathrm{Fe}_{83} \mathrm{~B}_{17}$ is nearly independent of the applied load, its $\Delta H_{c} / \Delta \sigma$ value is rather small.

The obtained data show that the eutectic alloy $\mathrm{Fe}_{83} \mathrm{~B}_{17}$ is the least sensitive to changes in temperature and applied load: the changes per unit stress of its coercivity $\Delta H_{c} / \Delta \sigma$, remanence $\Delta B_{r} / \Delta \sigma$ and permeability $\Delta \mu_{\max } / \Delta \sigma$ are comparatively small at both studied temperatures. The threshold (i.e. separating the unchanged and changed electronic structures) $\mathrm{Fe}_{84} \mathrm{~B}_{16}$ alloy is the most sensitive to applied load at room temperature, and its properties are the most sensitive to temperature as well. When the temperature decreases its sensitiveness to the load drops sharply.

\section{Uniaxial pressure effects}

The data obtained in the uniaxial pressure and the tensile experiments are in a good agreement. The magnetic susceptibility drops under pressure at all the temperatures studied, but the temperature dependence is not monotonic.

When lowering the temperature all the alloys showed an increasing value of $\chi^{-1} d \ln \chi / d P$ in the range $230-150 \mathrm{~K}$ (for alloy $\mathrm{Fe}_{84} \mathrm{~B}_{16}$ the increase was only small), a maximum at $110-140 \mathrm{~K}$, a decrease at $110-30 \mathrm{~K}$, and an abrupt growth at tem-

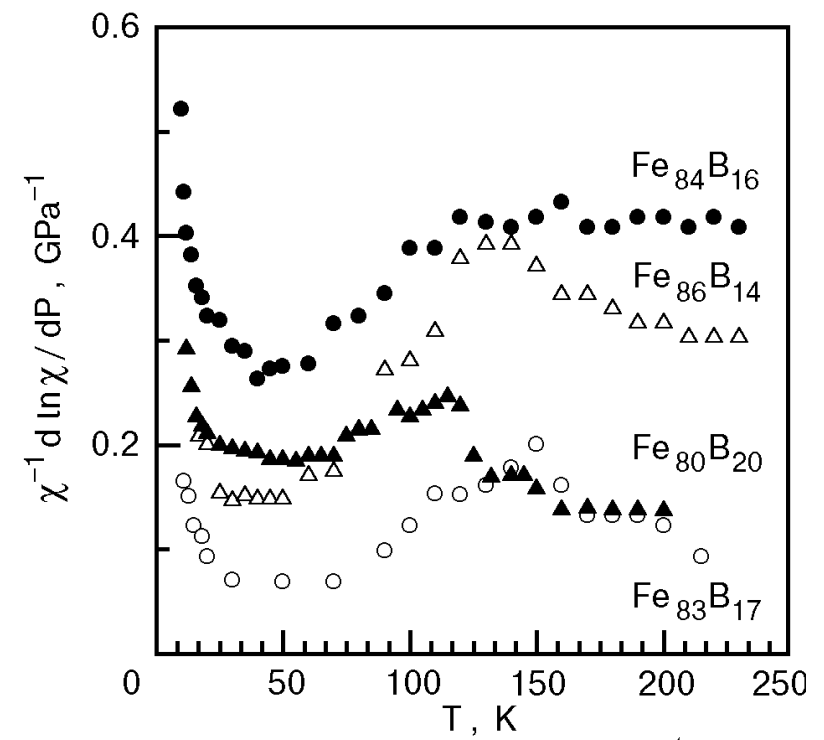

Fig. 5. The temperature dependence of the value $\chi^{-1} d \ln \chi / d P$ for amorphous $\mathrm{Fe}-\mathrm{B}$ alloys under the normal to the ribbons surface uniaxial pressure of $20 \mathrm{MPa}$. 
peratures lower than $30 \mathrm{~K}$ (Fig. 5). The $\chi^{-1} d \ln \chi / d P$ value was chosen for the analysis, because it is independent of the demagnetization factor: $\chi^{-1} d \ln \chi / d P=\chi_{\text {eff }}^{-1} d \ln \chi_{\text {eff }} / d P$. The main features of the temperature behavior of this value are determined by the behavior of $d \chi_{\mathrm{eff}} / d P$, because $\chi_{\text {eff }}$ comparatively slightly depends on the temperature.

Since the interatomic distances weakly change with temperature (the studied alloys possess invartype properties [9]), the presence of $\chi^{-1} d \ln \chi / d P$ maxima means a change in electronic structure. The growth of $\chi^{-1} d \ln \chi / d P$ at temperatures lower than $30 \mathrm{~K}$ is evidently connected with the formation of an asperomagnetic structure in this temperature region [7].

The smallest value of $\chi^{-1} d \ln \chi / d P$ corresponds to the eutectic composition $(x=17)$. This is in a good agreement with the concentration dependence of the failure stress of the studied alloys [4], as well as with our data on tension stress effects.

\section{Conclusion}

The results obtained confirm that the eutectic alloy $\mathrm{Fe}_{83} \mathrm{~B}_{17}$ possesses a special and most stable atomic and electronic structure with the highest local anisotropy. It has the lowest magnetic induction and permeability, and it is the least sensitive to applied load and decreasing temperature. The results are in good agreement with the idea of an extremely disordered structure of intercluster boundaries of eutectic alloy $\mathrm{Fe}_{83} \mathrm{~B}_{17}$, in which the clusters are supposed to have a quasicrystal structure [4].

The hypoeutectic $\mathrm{Fe}_{84} \mathrm{~B}_{16}$ alloy exhibits the highest value of magnetic permeability and the lowest remanence and coercivity. It is the most sensitive to the applied loads at room temperature, and its properties are also the most sensitive to changes in temperature. Therefore, it possesses the smallest local magnetic anisotropy and the least stable electronic structure.

\section{Acknowledgment}

The amorphous ribbons were let for the research by P. Duhaj (Institute of Physics of Slovak Academy of Sciences, Bratislava). Authors are thankful to P. Duhaj, E. D. Tabachnikova and A. S. Panfilov for collaboration.

\section{Dedication}

Authors dedicate this paper to the memory of Boris Ieremievich Verkin, Scientist and Organizer of Science, who has founded and for thirty years Directed the Institute for Low Temperature Physics and Engineering of the Academy of Sciences of Ukraine, where authors have good luck to work.

1. F. E. Luborsky, Amorphous Metallic Alloys, Butterworths, London (1983).

2. R. Hasegawa and R. Ray, J. Appl. Phys. 49, 4174 (1978).

3. R. Hasegawa, J. Magn. Magn. Mat. 100, 1 (1991).

4. V. Z. Bengus, P. Duhaj, E. B. Korol'kova, E. D. Tabachnikova, A. V. Golik, and S. I. Tarapov, Fiz. Nizk. Temp. 20, 1082 (1994) [Low. Temp. Phys. 20, 853 (1994)].

5. A. B. Beznosov, E. L. Fertman, V. A. Desnenko, V. Z. Bengus, and V. A. Ushakov, in: Novye Magnitnye Materialy Mikroelectroniki, Pt. 1, 212, MGU, Moskva (1998).

6. R. S. Iskhakov, M. M. Karpenko, G. V. Popov, and V. P. Ovcharov, Fiz. Met. Metalloved. 61, 265 (1986).

7. V. G. Bar'yakhtar, G. A. Takzei, A. B. Surzhenko, and M. V. Gavrilenko, Fiz. Tverd. Tela 34, 245 (1992).

8. S. V. Vonsovsky, Magnetism, Nauka, Moskva (1971).

9. K. Fukamichi, M. Kikuchi, S. Arakawa, and T. Masumoto, Solid State Commun. 23, 955 (1977). 